\title{
A survey on the impact of COVID-19 infection on menstrual cycle following second wave of COVID infection in a tertiary care centre in Mumbai
}

\author{
Niranjan N. Chavan', Manan Murlidhar Boob ${ }^{1 *}$, Sonam Simpatwar', \\ Ashwini Sakhalkar ${ }^{1}$, Neetika N. Chavan ${ }^{2}$
}

\author{
${ }^{1}$ Department of Obstetrics and Gynaecology, Lokmanya Tilak Municipal Medical College, Sion, Mumbai, \\ Maharashtra, India \\ ${ }^{2}$ Krishna Institute of Medical Sciences, KIMS, Karad, Maharashtra, India
}

Received: 13 December 2021

Revised: 07 January 2022

Accepted: 10 January 2022

*Correspondence:

Dr. Manan Murlidhar Boob,

E-mail: mananboob2296@gmail.com

Copyright: ( ) the author(s), publisher and licensee Medip Academy. This is an open-access article distributed under the terms of the Creative Commons Attribution Non-Commercial License, which permits unrestricted non-commercial use, distribution, and reproduction in any medium, provided the original work is properly cited.

\section{ABSTRACT}

Background: The COVID-19 pandemic has led to poor mental health measures, with emerging evidence suggesting gender differences with poorer outcomes in women. It has also introduced many acute stressors in life of many women and thus, led to worse outcomes. This may have unintended consequences for women's overall health and well-being, including disruptions to reproductive function as elevated stress is often associated with menstrual cycle irregularities. The objective of this study was to determine if and how the COVID-19 infection and its related stressors have impacted women's menstrual cyclicity.

Methods: An online survey was designed to capture self-reported information on menstrual cycle changes was distributed between April and September 2021. A total of 155 women who met stringent inclusion and exclusion criteria and completed the survey.

Results: Of the 155 respondents, more than half $(78 \%)$ reported changes in their menstrual cycles. These included changes in menstrual cycle length, the duration of menses, or changes in premenstrual symptoms.

Conclusions: By uncovering a trend in increased menstrual cycle irregularities during the second wave of the COVID-19 pandemic, this study contributes to our understanding of the implications that the pandemic may have on women's reproductive health.

Keywords: Menstrual cycle, COVID-19, Reproductive health

\section{INTRODUCTION}

In December 2019, a disease outbreak characterized by acute atypical respiratory symptoms was reported in the city of Wuhan, China. Chinese health officials identified a novel coronavirus (2019n-Cov) as the source of the outbreak, later named COVID-19 by the World health organization (WHO). ${ }^{1}$ By March 2020, COVID-19 had rapidly spread across 114 countries and amassed $>118,000$ confirmed cases triggering the WHO to declare
COVID-19 a pandemic. ${ }^{2}$ To control the spread of COVID-19, it was recommended to implement social distancing across the world, and to varying degrees countries issued stay-at-home orders, which included the closures of schools and nonessential businesses. ${ }^{3,4}$ Menstrual cyclicity is an indicator of reproductive function, which is susceptible to disruptions from stress, insomnia, depression and other systemic diseases. Menstrual cycle irregularities such as amenorrhea and changes in menstrual and premenstrual symptoms are 
often reported by women with mood disorders such as anxiety and depression, or by those facing acute life stressors..$^{5-7}$ The female reproductive system is subject to modulating influences of stress through the interconnected nature of the hypothalamic-pituitarygonadal (HPG) and hypothalamic-adrenal axes. Similarly, various systemic diseases such as thyroid disorders, diabetes, hypertension, etc. and infections such as tuberculosis have an impact on menstrual cycle regularity. This study was conducted to assess whether there are any changes in menstrual cycle as self-reported by the participants. There have been multiple studies which suggest a significant change in menstrual patterns due to the COVID pandemic, however, none of them have assessed the relation between COVID-19 infection with changes in patterns of menstruation. Thus, this study aims to bridge this gap.

\section{METHODS}

An online survey conducted from Lokmanya Tilak Municipal Medical College and General Hospital, Mumbai over the period from 22 April 2021 to 24 September 2021. Data collection was done online. The survey was answered online by 155 COVID-19 infected patients. We developed a survey to assess self-reported changes in menstrual cyclicity both during and after COVID-19 infection in patients. The survey questions were internally validated by the investigators.

\section{Statistical analysis}

All the parameters were studied and analysed on the basis of percentages. As this was a purely observational study, all parameters were analysed using descriptive statistics i.e. percentages and proportions were calculated and no statistical test was applied.

\section{Validation of questionnaire}

Questionnaire was prepared in English on surveyheart.com and its content validity was assessed by two co-investigators and then sent to patients via WhatsApp, Gmail and social media. All answers were collected and results were prepared from given answers.

\section{RESULTS}

Maximum participants, $100(64.5 \%)$, were from age group of 18-25 years. Other age groups had the following participants 26-35 years, 40 (28.38\%), 36-45 years, 10 (6.4\%), 46-50 years, $5(3.2 \%)$. Most of the participants were students, $120(77 \%)$. About 30 (19\%) were working women and the remaining were homemakers $5(3.2 \%)$. Majority of the women, $120(77 \%)$ in our survey were unmarried, whereas $25(16 \%)$ women were married. The participating women most commonly had a normal BMI, $95(61.3 \%)$. With $50(32.3 \%)$ women being overweight, around 5 women $(3.2 \%)$ each were underweight and obese. Around 75 women (48.4\%) were graduates and about 35 (22.6\%) women were post graduates.

Most of the women participating in the survey had regular menses, $110(71 \%)$.

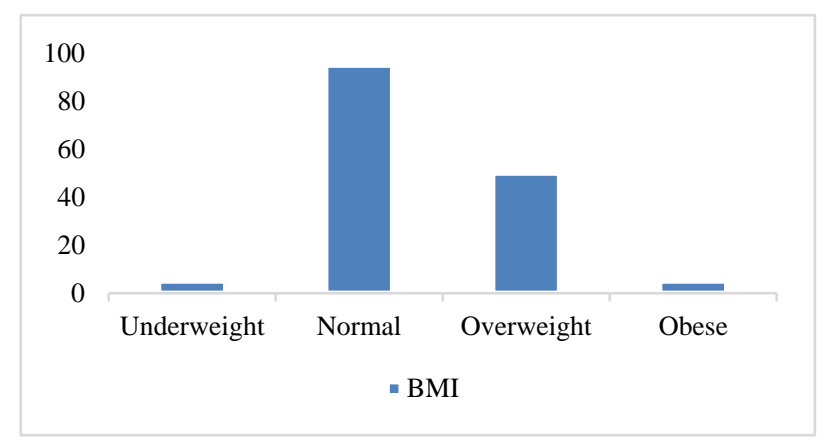

Figure 1: BMI.

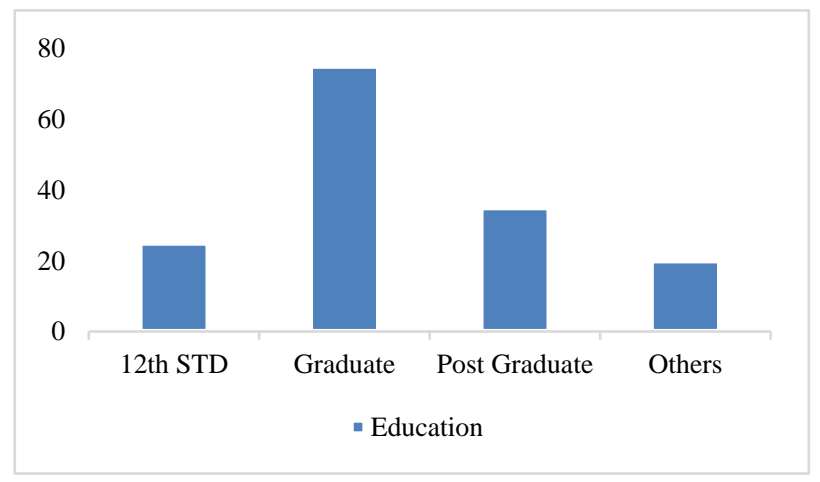

Figure 2: Educational status.

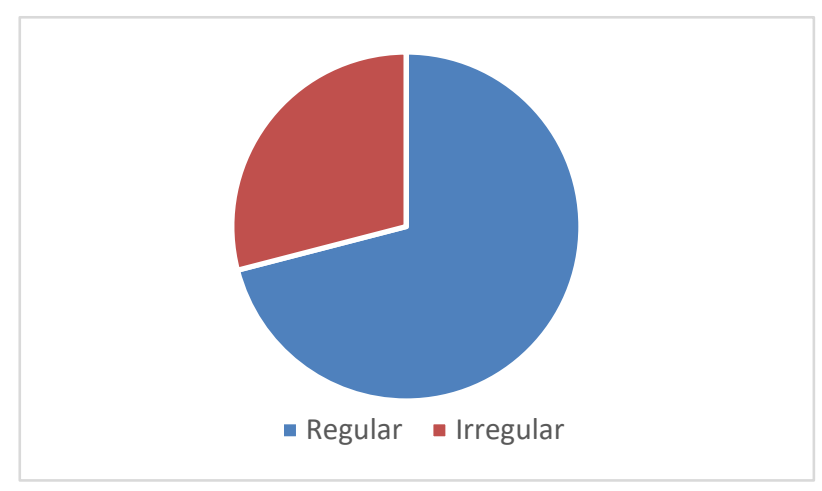

Figure 3: Regularity of menses.

About 29\% (45) women reported irregularity in menses. Around 100 (91\%) women were menstruating whilst having COVID-19 infection. Around 78 (78\%) women of the ones who had menses while they were infected with COVID-19 virus had irregular menses and the remaining $22(22 \%)$ women had regular menses. While around 99 $(64 \%)$ women had irregularity in menses after the COVID-19 infection, only 56 (29\%) women had regular menses. Majority of the women, $110(71 \%)$ had no other co-morbid conditions contributing to the irregularity in menses. As most women were vaccinated, either 
completely or partially, they had either asymptomatic or mild form of COVID-19 infection. Only 5 (3\%) women reported a severe form of infection. An overwhelming majority, $110(71 \%)$, of the women were vaccinated with both doses of COVID vaccine. Whereas, about 35 (23\%) women were partially vaccinated and $10(6 \%)$ women had not received a single dose of the vaccine. Most women, $125(81 \%)$ had received the COVISHIELD vaccine, whereas, about $20(13 \%)$ received the COVAXIN vaccine. Only $10(6 \%)$ women had received vaccines other than the above two.

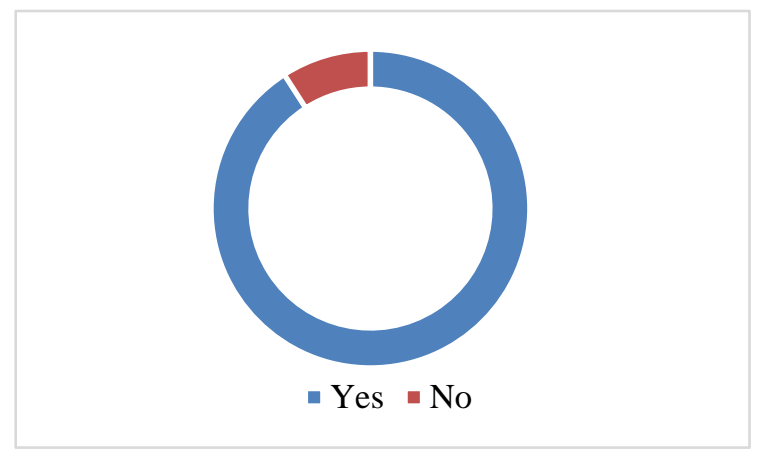

Figure 4: Did you get periods during COVID 19 infection?

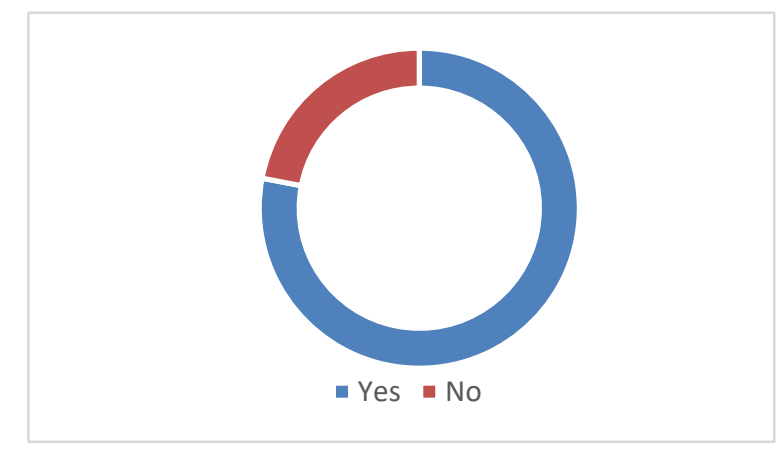

Figure 5: Were your menses irregular during COVID-19 Infection?

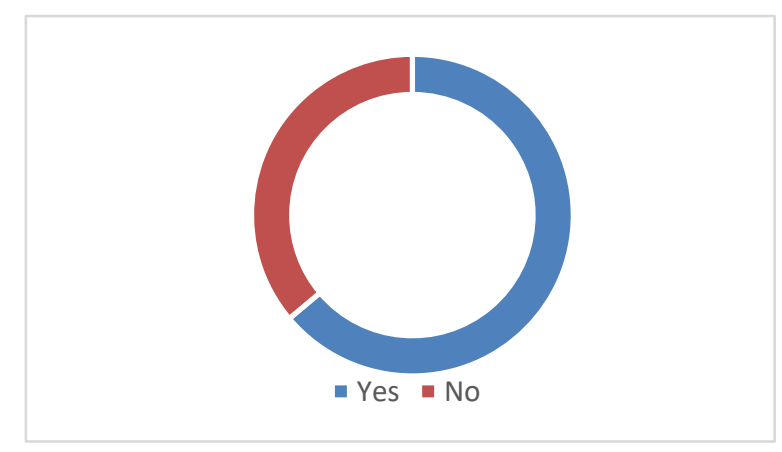

Figure 6: Were your menses irregular after COVID19 Infection?

\section{DISCUSSION}

The second wave of COVID-19 infection arrived in Mumbai in March 2021 and it was much quicker and faster spreading than the previous one. Around 4.5 million people were infected and about 89,000 lives were lost in Maharashtra state alone with Mumbai contributing about $10 \%$ to the death toll. The present study was an attempt to find any correlation between menstrual cycle regularity and the COVID-19 infection. This is one of the first studies to study the impact of COVID-19 infection on the menstrual regularity of women.

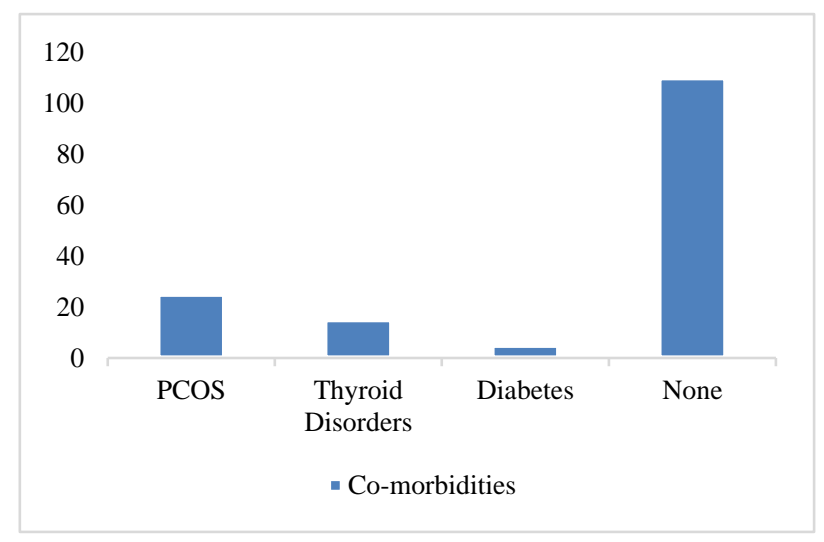

Figure 7: Do you have any underlying CO-MORBID condition(s)?

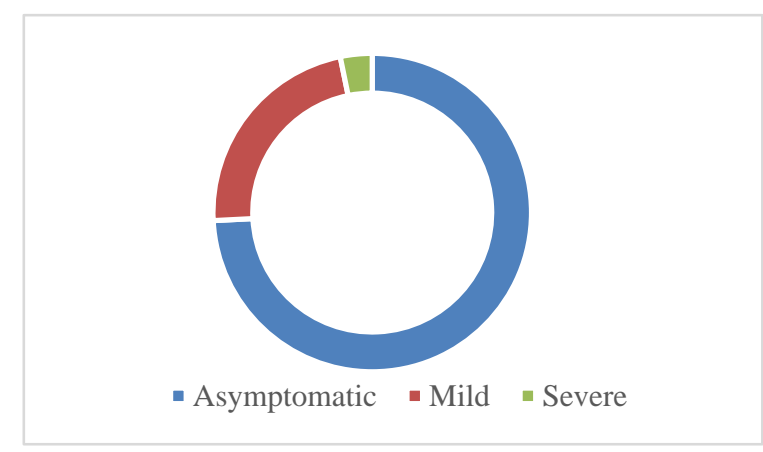

Figure 8: What was the severity of COVID-19 infection you had?

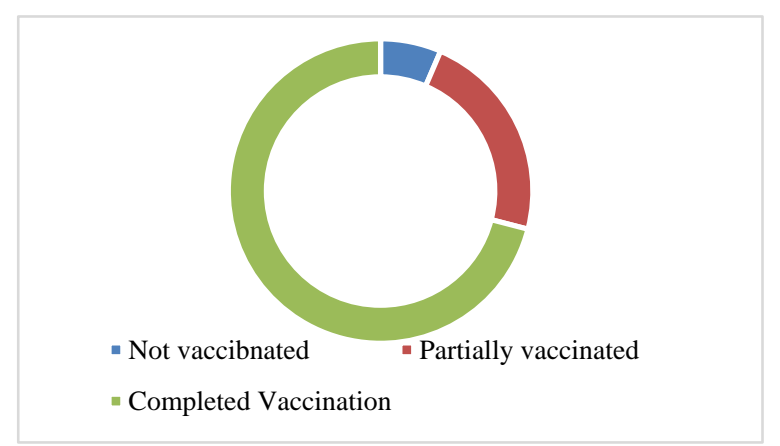

Figure 9: What is your COVID-19 Vaccination status?

It is well known that acute life stressors may cause women to experience irregularities in menstrual 
cyclicity. ${ }^{13-16}$ Previous studies also suggest that while being infected with COVID-19 is an acutely stressful situation, even among women who did not report significant stress related to the COVID-19 infection, alterations in menstrual cyclicity were common. ${ }^{17}$ Thus, COVID-19 infection, independently, may also cause changes in menstrual cycle. Further studies are required to evaluate this possible correlation. Most women reported menstrual irregularity after the infection with COVID-19 rather than during the infection. Majority of the women in our study had a mild form of disease and a majority of them were immunised. Thus, our study confirms the finding that, it is possible that a SARS-CoV2 vaccine could reduce severity of disease. ${ }^{8}$ There was no discernible association between disease severity and menstrual cycle irregularity in this particular study. The COVID-19 infection might have impact on the menstrual regularity in women irrespective of the associated stressors and thus, needs consideration.

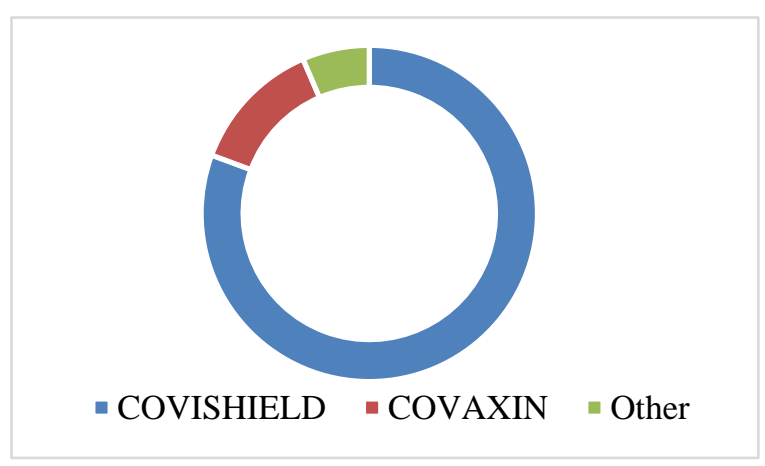

Figure 10: Which COVID-19 vaccine have you received?

\section{Limitations}

Limitations of this study include it being an online survey-based study with self-reported changes in menstrual cycle and no objective scale was used. Larger studies are required to validate our findings. There may be confounders such as increase in stress, hospitalisation as well as other disease pathology and comorbidities which might affect the outcomes of this study.

\section{CONCLUSION}

This study suggests that there may be significant changes in the menstrual cycle which are associated with COVID19 infection. As most of the subjects were vaccinated, the severity of the disease reported by them was possibly lesser, which confirms findings from other studies ${ }^{8}$. Further studies with larger sample size and objective scales are required to confirm this finding.

Funding: No funding sources Conflict of interest: None declared

Ethical approval: The study was approved by the Institutional Ethics Committee

\section{REFERENCES}

1. Coronaviridae study group of the international committee on taxonomy of viruses. The species severe acute respiratory syndrome-related coronavirus: Classifying 2019-ncov and naming it sars-cov-2. Nat Microbiol. 2020;5:536-44.

2. WHO director-general's opening remarks at the media briefing on COVID-19-11 march 2020. Available at: https://www.who.int/director-general/ speeches/detail/who-director-general-s-openingremarks-at- the-media-briefing-on-COVID-19-11march-2020. Accessed on 1 July 2020.

3. Moreland A, Herlihy C, Tynan MA. Timing of state and territorial COVID-19 stay-at-home orders and changes in population movement-United States, March 1-May 31, 2020. MMWR Morb Mortal Wkly Rep. 2020;69:1198-203.

4. WHO coronavirus disease (COVID-19) dashboard. Available at: https://covid19.who.int. Published 2021. Accessed on 20 November 2020.

5. Marroquin B, Vine V, Morgan R. Mental health during the COVID-19 pandemic: Effects of stay-athome policies, social distancing behavior, and social resources. Psychiatry Res. 2020;293:113419.

6. Tull MT, Edmonds KA, Scamaldo KM, Richmond JR, Rose JP, Gratz KL. Psychological outcomes associated with stay-at-home orders and the perceived impact of COVID-19 on daily life. Psychiatry Res. 2020;289:113098.

7. Salari N, Hosseinian-Far A, Jalali R. Prevalence of stress, anxiety, depression among the general population during the COVID-19 pandemic: A systematic review and meta-analysis. Global Health. 2020;16:57.

8. Hodgson SH, Mansatta K, Mallett G, Harris V, Emary KRW, Pollard AJ. What defines an efficacious COVID-19 vaccine? A review of the challenges assessing the clinical efficacy of vaccines against SARS-CoV-2. Lancet Infect Dis. 2021;21(2): e26-35.

9. Ozimek N, Velez K, Anvari H, Butler L, Goldman $\mathrm{KN}$, Woitowich NC. Impact of stress on menstrual cyclicity during the COVID-19 pandemic: a survey study. J Women's Heal. 2021;2(5):1-7.

10. Takmaz T, Gundogmus I, Okten SB, Gunduz A. The impact of COVID-19-related mental health issues on menstrual cycle characteristics of female healthcare providers. J Obstet Gynaecol Res. 2021;47(9):32419.

11. Demir O, Sal H, Comba C. Triangle of COVID, anxiety and menstrual cycle. J Obstet Gynaecol (Lahore). 2021;41(8):1257-61.

12. Male V. Menstrual changes after COVID-19 vaccination. BMJ. 2021;374:1-2.

13. Reese MA, Usta J, Gupta J, Ettinger AS. Assessment of reproductive health and violence against women among displaced Syrians in Lebanon. BMC Womens Health. 2014;14:25. 
14. Li XH, Qin L, Hu H. Influence of the wenchuan earthquake on self-reported irregular menstrual cycles in surviving women. Gynecol Endocrinol. 2011;27:706-10.

15. Hannoun AB, Nassar AH, Usta IM, Zreik TG, Abu Musa AA. Effect of war on the menstrual cycle. Obstet Gynecol. 2007;109:929-32.

16. Almeida M, Shrestha AD, Stojanac D, Miller LJ. The impact of the COVID-19 pandemic on women's mental health. Arch Womens Ment Health. 2020; 23:741-8.
17. Ozimek N, Velez K, Anvari H, Butler L, Goldman $\mathrm{KN}$, Woitowich NC. Impact of stress on menstrual cyclicity during the COVID-19 pandemic: a survey study. J Women's Heal. 2021;5(3):1-7.

Cite this article as: Chavan NN, Boob MM, Simpatwar S, Sakhalkar A, Chavan NN. A survey on the impact of COVID-19 infection on menstrual cycle following second wave of COVID infection in a tertiary care centre in Mumbai. Int J Reprod Contracept Obstet Gynecol 2022;11:491-5. 